\title{
Erratum to: Dual myeloperoxidase-antineutrophil cytoplasmic antibody- and antiglomerular basement membrane antibody-positive cases associated with prior pulmonary fibrosis: a report of four cases
}

Kimimasa Nakabayashi $\cdot$ Yasunori Fujioka $\cdot$ Toshihiko Nagasawa

Tsuneo Kimura Kaoruko Kojima Y Yoshihiro Arimura $\cdot$ Akira Yamada

Published online: 29 June 2011

(C) Japanese Society of Nephrology 2011

Erratum to: Clin Exp Nephrol (2011) 15:226-234

DOI 10.1007/s10157-010-0390-0

The correct name of the sixth author should be given as

Yoshihiro Arimura, not Yasuhiro Arimura.

The online version of the original article can be found under doi:10.1007/s10157-010-0390-0.

K. Nakabayashi ( $\square) \cdot$ T. Nagasawa · T. Kimura ·

Y. Arimura $\cdot$ A. Yamada

First Department of Internal Medicine,

Kyorin University School of Medicine,

6-20-2 Shinkawa, Mitaka, Tokyo 181-8611, Japan

e-mail: kiminaka@krd.biglobe.ne.jp

Y. Fujioka $\cdot$ K. Kojima

Department of Pathology,

Kyorin University School of Medicine,

Mitaka, Japan 